\title{
Gas-Chromatographic Analysis of Major Volatile Compounds Found in Traditional Fruit Brandies from Transylvania, Romania
}

\author{
Teodora Emilia RUSU COLDEA, Carmen SOCACIU*, Maria PÂRV, Dan VODNAR \\ ${ }^{1}$ University of Agricultural Sciences and Veterinary Medicine, 3-5 Mănăşstur Street, 400372, Cluj- \\ Napoca, Romania; csocaciudac@gmail.com (*corresponding author)
}

\begin{abstract}
In the current study, the major volatile compounds from three categories of traditional fruit brandies (plum, apple and pear) were characterized by gas-chromatography (GC-FID). There were collected 26 samples from different locations of Transylvania (Romania), all made by traditional technologies involving fermentation in barrels and distillation in copper stills. The major volatile compounds, besides ethanol, identified and quantified were: acetaldehyde, ethyl acetate, methanol, 1-propanol, 2-butanol, iso-butylic alcohol, alcool amyl active, iso-amylic alcohol, 1-butanol and furfural. For each type of brandy, positive but no significant correlations between methanol and furfural concentrations in plum and apple brandy were noticed. To evaluate the differences in composition regarding the geographical origin of plum brandies and to analyze the composition of plum, apple and pear brandies it has been compared the mean values (MVP, MVA and MVPe) obtained for each volatile. For plum brandies it has been observed differences among the mean values of each volatile, in samples originating from counties Cluj, Bistrița-Năsăud and Maramureş. For methanol, acetaldehyde and 1-propanol the MVP Cluj values were significantly higher than MVP Bistrița-Năsăud. For iso-butylic alcohol, amyl active alcohol, iso-amylic alcohol the MVP Cluj values were significantly higher than for Bistrița-Năsăud and Maramureş, while for ethyl acetate and furfural the MVP Bistrița-Năsăud were significantly higher than MVP Cluj and MVP Maramureș. When compared the mean values of volatiles in plum $v s$ apple $v$ s pear brandies, for ethyl acetate, methanol, 2-butanol, 1-propanol and 1-butanol, the MVPe values were significantly higher than MVA, for furfural, amyl active and iso-amylic alcohols, while for acetaldehyde the MVPe values were significantly higher than MVP. Methanol represented the major volatile component, characteristic to fruit brandies, released by enzymatic degradation of methoxylated pectins. Therefore, this molecule can be considered not only a parameter of distillate safety but also an indicator of natural origin of distillate and traditional processing.
\end{abstract}

Keywords: ANOVA, correlation coefficient, direct injection, Duncan, GC-FID

\section{Introduction}

Romania has an old and rich tradition in fruit growing and traditional distilled beverages, their assortment being enlarged significantly in the last decades. The traditional method used to obtain fruit brandies is the distillation of fruit pulp in copper stills with open fire, maturing and conditioning in oak barrels (Pomohaci, 2002).

The most important Romanian traditional fruit distillate is plum brandy known as "țuica" (denominated by Romanian legislation), obtained exclusively from plums and "pălinca", a brandy obtained from a mixture of fruits.

The fruit fermentation is made in wood barrels or in stainless steel recipients. Distillation is made in copper stills under open fire or in distillation installation. Usually distillation is repeated twice, the final alcoholic concentration of these products is between 24 to $86 \% \mathrm{v} / \mathrm{v}$, for "țuica" and 40-70\% v/v, for "pălinca". In Romania, there are different types of fruit brandies and according to the region, it can be find different alcoholic concentrations of these drinks. For example, in the south of the country "țuica" has values around 30\% v/v (single distillation), but in Transylvania region, its values are around 50\% v/v (in most cases obtained by double distillation process).

No matter the production process applied, the flavor and taste of these traditional beverages may be an indicator of the fruit or fruits used as raw materials.

The storage and the maturing are achieved in wood barrels, stainless steel or glass recipients for at least three months. The yellow or gold-yellow color of these traditional distillates can be obtained exclusively by maturing in wood barrels (the most used being the oak barrels), without any colorants or pure alcohol of industrial origin adding.

The preparation and fermentation of raw material, distillation technology and maturation are main factors responsible for the specific bouquet of fruit brandies.

Some of volatiles found in fruit distillates, such as methanol, furfural, iso-butylic alcohol and acetaldehyde have toxic potential. This is the reason why the European Commission established a maximum admissible value for methanol in fruit brandies being $1200 \mathrm{mg} / 100 \mathrm{ml}$ anhydrous alcohol (anh. alc.). In the case of ethyl alcohol of agricultural origin, the limits of these toxic compounds are 
110

more restrictive (acetaldehyde-maximum $0.5 \mathrm{mg} / 100 \mathrm{ml}$ anh. alc.; methanol-30 mg/100 ml anh. alc.; furfural-not detectable) (REG.110/2008).

Beside ethanol, from all major volatile substances, methanol has the highest amount in fruit distillates. In most cases, it is ingested by the consumers in low doses when consuming alcoholic beverages.

Methanol is found in high amounts in raw distillate (resulted after the first distillation) as well in the final brandy. This happen because the methanol concentration is directly related to the quantity of pectins present in fruits (peels, seeds) which are methoxylated during fruit ripening. By the effect of pectases, the demethylation can occur and methanol is released together with pectic acid and pectol.

After re-distillation, methanol is concentrated in overhead, and then it can be reduced and the final distillate to contain concentration in accordance with the maximum admissible levels. It is formed when pectic substances hydrolyse under the influence of some pectolytic enzymes (especially, pectin methyl esterase). The distillates produced from different fermented fruits have generally higher amount of methanol in comparison with those obtained by other techniques (ethyl alcohol from cereal processing), due to the degradation of methoxylated pectins found in fruit flesh. This is the reason why methanol is present in fruit distillates and may indicate the origin of raw material (Moales et al., 2010) or the authenticity of a natural fruit brandy vs a brandy containing fruit synthetic essence and refined ethanol (Nikićević and Tešević, 2005).

Furfural, derived from fruit carbohydrates, contribute to the aroma and flavor of fruit distillates. By ingestion, furfural may be toxic to human organism, inducing pain, sore throat, diarrhea, vomiting and headache. Furfural can have harmful effects by inhibition of enzymatic substrates in fermentation process (Modig et al., 2002).

The classical distillation method (which integrates the copper still) with direct heating can create harmful effects such as mashes with a burnt-bitter taste caused by furfural formation. This effect is hardly removable (Berglund, 2004). The caramel color can be also an explanation of furfural presence (Quesada Granados et al.,1996).

Acetaldehyde is a result of ethanol dehydrogenation and can occur during fermentation, as a minor volatile. It has toxic effects when ingested, causing diarrhea, dizziness, nausea and vomiting.

Significant data were reported the last years, regarding the volatiles in traditional brandies from Central and East, South-East European countries (Tab. 1).

Slivovice, another name of a traditional plum brandy, togheter with other distilled beverages were characterized and classified by total luminescence and synchronous fluorescence spectroscopy (Tóthová et al., 2008).

Few data about the quality of Romanian fruit distilates are reported. Recently, the chemical analysis of distillated beverages has been made by Beceanu and Nicula (2009); Beceanu et al. (2010) for some traditional and commercial distilled alcoholic beverages made in Romania.

In this study, the aim was to fingerprint and quantify the specific volatile compounds found in three categories of home-made fruit brandies. The brandies were made by the distillation of different fermented fruits of plum, apple and pear.

Tab. 1. The reported volatiles identified in different types of distillates and the applied techniques

\begin{tabular}{|c|c|c|c|}
\hline Type of distillate & The volatile compound determinated & $\begin{array}{l}\text { Techniques } \\
\text { of analysis }\end{array}$ & References \\
\hline Traditional fruit distillates & Methanol & GC-MS & Moales et al. (2010) \\
\hline Plum brandy & Methanol & GC-FID & $\begin{array}{l}\text { Nikićević and } \\
\text { Tešević (2005) }\end{array}$ \\
\hline $\begin{array}{l}\text { Commercial brandies } \\
\text { and wine spirits }\end{array}$ & Furfural & HPLC & $\begin{array}{l}\text { Quesada Granados } \\
\text { et al. (1996) }\end{array}$ \\
\hline Slivovitz (plum brandy) & $\begin{array}{l}\text { 1-propanol, 2-propanol, ethyl acetate, } \\
\text { 2-methyl-1-propanol etc. }\end{array}$ & $\begin{array}{l}\text { GC-MS and } \\
\text { SPME-GC-MS }\end{array}$ & Korhonova et al. (2006) \\
\hline Apple brandy & Aroma fraction (furfural, ethyl acetate etc.) & $\begin{array}{l}\text { HRGC-FID and } \\
\text { HRGC-MS }\end{array}$ & Versini et al. (2009) \\
\hline Whey spirit & $\begin{array}{l}\text { Major volatile compounds (acetaldehyde, ethyl acetate, iso- } \\
\text { amyl alcohol, 1-propanol, 2-butanol, furfural, 1-butanol, etc.) }\end{array}$ & GC-FID & Dragone et al. (2009) \\
\hline $\begin{array}{l}\text { Hellenic alcoholic beverages } \\
\text { from white grapes }\end{array}$ & $\begin{array}{l}\text { Major volatile compounds (methanol, acetaldehyde, } \\
\text { 2-butanol, 1-propanol, 1-butanol, ethyl acetate, etc.) }\end{array}$ & $\begin{array}{l}\text { gas/liquid } \\
\text { chromatography }\end{array}$ & $\begin{array}{l}\text { Christopoulou- } \\
\text { Gerogiannaki et al. (2007) }\end{array}$ \\
\hline Brandy de Jerez & $\begin{array}{l}\text { Aromatic profile (furfural, 1-propanol, } \\
\text { iso-butanol, 1-butanol, etc.) }\end{array}$ & GC-MS & Martínez Montero (2006) \\
\hline Plum brandy & Methanol, higher alcohols, esters, ethyl acetate, benzaldehyde & GC & Popović et al. (2009) \\
\hline Plum brandy & Methanol, ethanol, higher alcohols & GC-MS and GC-FID & Tešević et al. (2005) \\
\hline $\begin{array}{l}\text { Drenja (alcoholic beverage } \\
\text { made from cornelian cherry) }\end{array}$ & $\begin{array}{l}\text { Specific aroma compounds (alcohols, esters, monoterpene, } \\
\text { lactones, volatile phenols, acetal compounds) }\end{array}$ & GC-MS & Tešević et al. (2009) \\
\hline Turkish Raki beverage & $\begin{array}{l}\text { Methanol, acetaldehyde, ethyl acetate, 1-propanol, } \\
\text { 2-butanol, 1-pentanol, ethyl lactate, etc. }\end{array}$ & GC-MS & Ertan Anli et al. (2007) \\
\hline
\end{tabular}




\section{Materials and methods}

\section{Provenience of beverage samples}

The samples were collected, directly from the producer; 26 samples of homemade fruit distillates (plum brandy, apple brandy and pear brandy) processed between 2008, October and 2010, September, originating from different counties of Transylvania (Romania) such as: Maramureş, Cluj, Bistrița-Năsăud, Alba, Bihor (Tab. 2).

\section{Alcohol concentration and the relative density}

The determination of the alcoholic concentration and relative density were made by the electronic densitometer type DDM2911, with digital display and measuring cell connected to an incorporated temperature regulator, made by Rudolf Research Analytical, series: 2045, measuring domain: $0-3 \mathrm{~g} / \mathrm{cm}^{3}$. The density was displayed with 5 decimals and alcoholic concentration with 2 decimals.

\section{Volatile compounds analysis}

Analysis of major volatile compounds in fruit brandies was adapted after the EU reference method for volatile compounds. For the determination of the major volatile compounds, the samples were injected directly into the gas chromatograph column, from a GC-FID Agilent Tehnologies Gas chromatograph, 6850A, without preliminary treatment.

Each sample was injected twice in the GC-FID. One microliter from each sample was introduced on the capillary chromatography column ZB-WAX plus (characteristics: $60 \mathrm{~m}$ length, $0.25 \mathrm{~mm}$ diameter, $0.25 \mu \mathrm{m}$ film thickness, stationary phase: cross linked polyethylene glycol) produced by Zebron Company. Inside the oven, the initial temperature was $35^{\circ} \mathrm{C}$. The injector temperature was $240^{\circ} \mathrm{C}$-automatic injection; the carrier gas was helium and the detector (FID) temperature was $250^{\circ} \mathrm{C}$ (Tab. 3). The total time analysis was $30.63 \mathrm{~min}$.

The main components (methanol, acetaldehyde, ethyl acetate, 1-propanol, 2-butanol, iso-butilic alcohol, amyl active alcohol, iso-amylic alcohol, 1-butanol), were identified by comparing their retention times with those of authentic compounds.

For quantitative evaluation it was applied the internal standard method, with a known amount of 3-pentanol, as internal standard (IS). As such, a solution containing 0.1 $\mathrm{ml} 3$-pentanol was added to $10 \mathrm{ml}$ of every each sample.

For all volatiles, the quantitative evaluation was based on automatic calculation, based on peak area integration, while for furfural the integration was done, manually.

\section{Chemicals and reagents}

All used chemicals (ethanol, acetaldehyde, methanol, propanol, 1-butanol, 2-butanol, iso-butylic alcohol, isoamylic alcohol, amyl active alcohol, ethyl acetate, 3-pentanol) with purity over 99\% were provided by Merck and Sigma Aldrich Company.
Tab. 2. The location, type and codification of the beverage samples

\begin{tabular}{|c|c|c|c|c|}
\hline \multirow[t]{2}{*}{ Code } & \multirow{2}{*}{$\begin{array}{c}\text { Type of fruit } \\
\text { distillate }\end{array}$} & \multicolumn{2}{|c|}{ Producer localization } & \multirow{2}{*}{$\begin{array}{c}\text { Production } \\
\text { year }\end{array}$} \\
\hline & & Couty & Village & \\
\hline P1 & Plum brandy & Cluj & Tioltiur & 2009 \\
\hline P2 & Plum brandy & Cluj & Petrești & 2009 \\
\hline P4 & Plum brandy & Cluj & Ciucea & 2009 \\
\hline P6 & Plum brandy & Cluj & Morlaca & 2009 \\
\hline P17 & Plum brandy & Cluj & Bârlea & 2007 \\
\hline P18 & Plum brandy & Cluj & Negreni & 2009 \\
\hline P3 & Plum brandy & Bistrița-Năsăud & Beclean & 2009 \\
\hline P12 & Plum brandy & Bistrița-Năsăud & Năsăud & 2009 \\
\hline P13 & Plum brandy & Bistrița-Năsăud & Rebrişoara & 2009 \\
\hline P14 & Plum brandy & Bistrița-Năsăud & Salva & 2009 \\
\hline P15 & Plum brandy & Bistrița-Năsăud & Runc & 2009 \\
\hline P16 & Plum brandy & Bistrița-Năsăud & Feldru & 2010 \\
\hline P5 & Plum brandy & Maramureş & Seini & 2008 \\
\hline P7 & Plum brandy & Maramureş & Săliştea de Sus & 2009 \\
\hline P8 & Plum brandy & Maramureş & Vişeu de Jos & 2009 \\
\hline P9 & Plum brandy & Maramureş & Vişeu de Sus & 2009 \\
\hline P10 & Plum brandy & Maramureş & Moisei & 2009 \\
\hline P11 & Plum brandy & Maramureș & Leordina & 2009 \\
\hline M19 & Apple brandy & Bistrița-Năsăud & Coşbuc & 2010 \\
\hline M20 & Apple brandy & Alba & Ocoliş & 2008 \\
\hline M21 & Apple brandy & Cluj & Câțcău & 2009 \\
\hline M22 & Apple brandy & Bihor & Tulca & 2008 \\
\hline PE23 & Pear brandy & Cluj & Negreni & 2009 \\
\hline PE24 & Pear brandy & Maramureş & Şişeşti & 2006 \\
\hline PE25 & Pear brandy & Bistrița-Năsăud & Runc2 & 2009 \\
\hline PE26 & Pear brandy & Cluj & Aiton & 2008 \\
\hline
\end{tabular}

Tab. 3. The temperature program used for the GC-FID analysis of all types of fruit brandies

\begin{tabular}{cccc}
\hline Steps & Rate & Final temperature & Final time \\
\hline 1 & $12^{\circ} \mathrm{C} / \mathrm{min}$ & $35-58^{\circ} \mathrm{C}$ & $4 \mathrm{~min}$ \\
2 & $3^{\circ} \mathrm{C} / \mathrm{min}$ & $58-85^{\circ} \mathrm{C}$ & $0 \mathrm{~min}$ \\
3 & $30^{\circ} \mathrm{C} / \mathrm{min}$ & $155^{\circ} \mathrm{C}$ & $3 \mathrm{~min}$ \\
4 & $200^{\circ} \mathrm{C} / \mathrm{min}$ & $230^{\circ} \mathrm{C}$ & $5 \mathrm{~min}$ \\
\hline
\end{tabular}

\section{Statistical analysis}

The results obtained from the individual experiments were used to calculate the mean values for plum, apple and pear brandy samples. Analysis of variance (ANOVA) and Duncan's multiple range tests were performed to analyse the results. Significance of differences was defined at the $5 \%$ threshold $(\mathrm{P}<0.05)$. All statistical analysis was carried out using Graph Pad Version 4.0 (Graph Pad Software Inc; San Diego, CA, USA).

\section{Results and discussion}

Ethanol concentration based on relative density determination

The values obtained for the alcoholic concentration based on relative density measurement were represented 
112

in Tab. 4. All ethanol concentrations were calculated from relative density by specific formulas (not shown).

The mean value of ethanol concentration in plum brandies was $51.21 \%$, while $50.24 \%$ for apple brandy. In pear brandy the mean value was $45.05 \%$, significantly inferior $(\mathrm{P}<0.05)$.

\section{GC-FID analysis}

The GC-FID chromatograms of two representative samples (P7 and M20) are presented in Fig. 1 A and B. The major peak corresponding to ethanol $\left(t_{R}=8.11 \mathrm{~min}\right)$ was eliminated, in order to illustrate the other components better.

The main volatile compounds were identified by their retention times and by comparison with pure standards. The internal standard used in all cases was 3-pentanol (peak 7). Based on the peak areas, for each sample it was calculated the concentration of each component, expressed as $\mathrm{mg} / 100 \mathrm{ml}$ anhydrous alcohol (anh. alc.). The individual
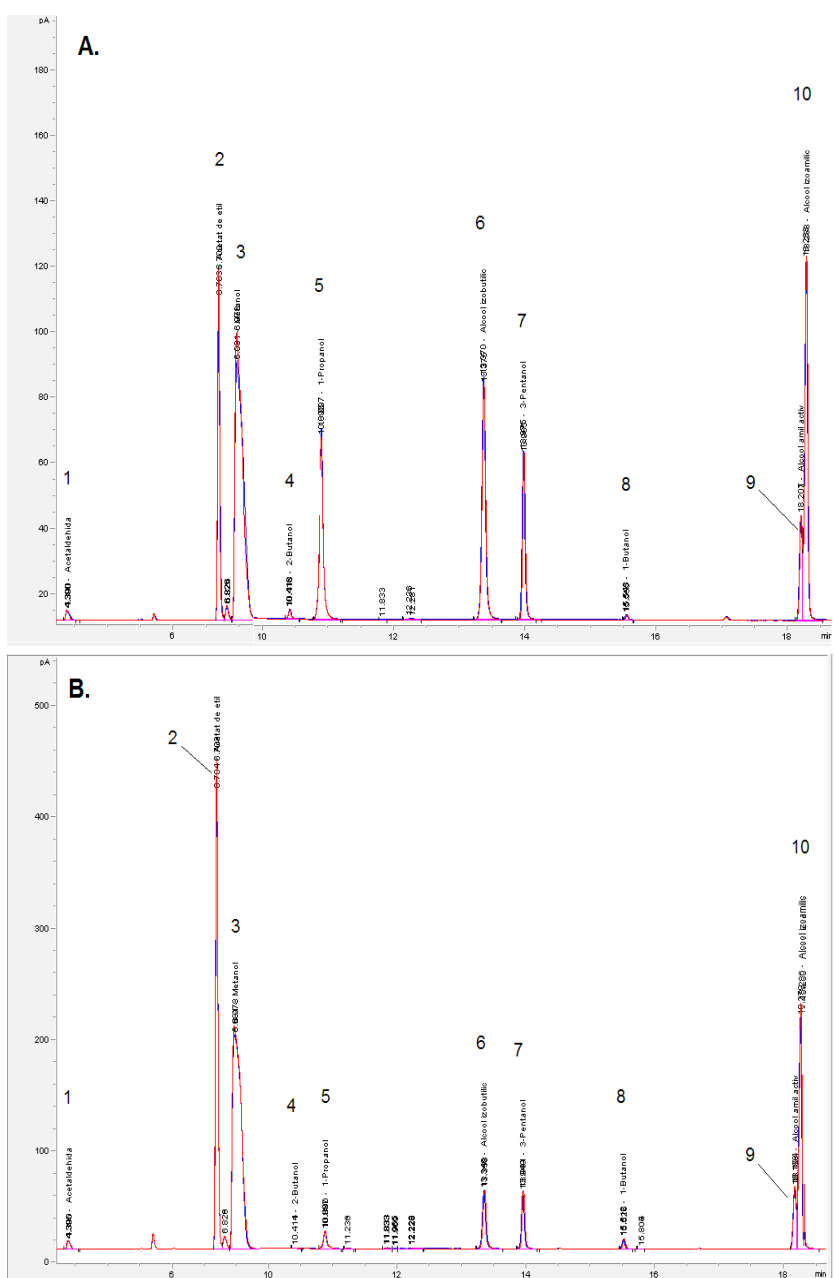

Fig. 1. The GC-FID chromatogram of major volatile compounds in sample P7 (A) and sample M20 (B). Peak identification: 1=Acetaldehyde; $2=$ =thyl acetate; $3=$ Methanol; 4=2-Butanol; 5=1-Propanol; 6=Iso-butylic Alcohol; 7=3-Pentanol (IS); 8=1Butanol; 9=Amyl Active Alcohol; 10=Iso-amylic Alcohol and mean values for each volatile found in plum (MVP), apple (MVA) and pear (MVPe) brandies are presented in Tab. 4-7.

For plum brandies (Tab. 5) it has been observed differences among the mean values (MVP) of each volatile, in plum brandies originating from 3 counties, Cluj, BistrițaNăsăud and Maramureş, For acetaldehyde and 1-propanol the MVP Cluj values were significantly higher than MVP Bistrița-Năsăud. Also, methanol MVP from Cluj were significantly higher $(\mathrm{P}<0.05)$ than MVP Maramureş and Bistrița-Năsăud. As an explanation for the presence of acetaldehyde can be the fact that aldehydes, especially acetaldehyde or ketones, are produced during in marc fermentation (Geroyiannaki et al., 2007; Garcia Llobodanin, 2008), their concentration level being higher if the copper still was not utilized properly or because of their higher

Tab. 4. Ethanol concentration, determined from relative density

\begin{tabular}{|c|c|c|}
\hline $\begin{array}{l}\text { Sample } \\
\text { code }\end{array}$ & $\begin{array}{c}\text { Alcoholic concentration } \\
(\% \mathrm{v} / \mathrm{v})\end{array}$ & $\begin{array}{l}\text { Relative density } \\
\left(\mathrm{g} / \mathrm{cm}^{3}\right)\end{array}$ \\
\hline P1 & 48.37 & 0.93330 \\
\hline P2 & 50.5 & 0.92916 \\
\hline P3 & 48.31 & 0.93342 \\
\hline P4 & 48.03 & 0.93395 \\
\hline P5 & 52.32 & 0.92551 \\
\hline P6 & 49.76 & 0.93061 \\
\hline P7 & 49.34 & 0.93142 \\
\hline P8 & 53.03 & 0.92406 \\
\hline P9 & 49.24 & 0.93162 \\
\hline P10 & 54.56 & 0.92089 \\
\hline P11 & 51.95 & 0.92626 \\
\hline P12 & 52.5 & 0.92514 \\
\hline P13 & 57.29 & 0.91506 \\
\hline P14 & 55.45 & 0.91902 \\
\hline P15 & 45.77 & 0.93815 \\
\hline P16 & 54.22 & 0.9216 \\
\hline P17 & 50.8 & 0.92859 \\
\hline P18 & 50.29 & 0.92956 \\
\hline MV & $51.21 \mathrm{a}$ & 0.93 \\
\hline $\mathrm{SD}$ & 2.96 & 0.01 \\
\hline M19 & 50.19 & 0.92976 \\
\hline M20 & 52.76 & 0.92461 \\
\hline M21 & 47.94 & 0.93411 \\
\hline M22 & 50.06 & 0.93003 \\
\hline MV & $50.24 a$ & 0.93 \\
\hline SD & 1.97 & 0.00 \\
\hline PE23 & 48.16 & 0.9337 \\
\hline PE24 & 44.89 & 0.93974 \\
\hline PE25 & 47.02 & 0.93585 \\
\hline PE26 & 40.12 & 0.94784 \\
\hline MV & $45.05 b$ & 0.94 \\
\hline $\mathrm{SD}$ & 3.55 & 0.01 \\
\hline
\end{tabular}

MV-mean value; SD-standard deviation. The values with different superscript letters $(\mathrm{a}, \mathrm{b}, \mathrm{c})$ are significantly different $(\mathrm{P}<0.05)$ 
Tab. 5. The major volatile compounds found in plum brandy as determined by gas-chromatography (GC-FID)

\begin{tabular}{|c|c|c|c|c|c|c|c|c|c|c|}
\hline \multirow[b]{2}{*}{ Sample code } & \multicolumn{10}{|c|}{ Volatile compound (mg/100 ml anhydrous alcohol) } \\
\hline & Acetaldehyde & $\begin{array}{c}\text { Ethyl } \\
\text { Acetate }\end{array}$ & Methanol & Furfural & 2-Butanol & 1-Propanol & $\begin{array}{c}\text { Iso-butylic } \\
\text { Alcohol }\end{array}$ & 1-Butanol & $\begin{array}{c}\text { Amyl Active } \\
\text { Alcohol }\end{array}$ & $\begin{array}{c}\text { Iso-amylic } \\
\text { Alcohol }\end{array}$ \\
\hline P1 & 13.70 & 223.26 & 1077.59 & 1.92 & 7.53 & 32.41 & 97.34 & 17.07 & 53.29 & 239.24 \\
\hline P2 & 12.99 & 691.67 & 1266.97 & 1.41 & 18.31 & 95.10 & 36.41 & 19.47 & 17.94 & 82.13 \\
\hline P4 & 5.25 & 125.55 & 954.31 & 1.56 & 0.66 & 72.29 & 128.81 & 1.95 & 54.65 & 219.96 \\
\hline P6 & 14.66 & 226.51 & 1244.35 & 2.28 & 8.65 & 175.09 & 49.30 & 11.59 & 20.71 & 73.22 \\
\hline P17 & 31.45 & 307.00 & 1100.76 & 3.58 & 0.17 & 132.81 & 42.24 & 6.78 & 22.73 & 93.62 \\
\hline P18 & 12.62 & 131.05 & 953.69 & 6.38 & 9.98 & 61.54 & 102.84 & 7.50 & 42.52 & 157.96 \\
\hline MVP Cluj & $15.11 \mathrm{a}$ & $284.17 \mathrm{~b}$ & $1099.61 \mathrm{a}$ & $2.86 \mathrm{~b}$ & $7.55 b$ & $94.87 \mathrm{a}$ & $76.16 a$ & $10.73 b$ & $35.31 \mathrm{a}$ & $144.36 a$ \\
\hline $\mathrm{SD}$ & 8.68 & 210.82 & 135.54 & 1.89 & 6.71 & 51.76 & 38.43 & 6.64 & 16.87 & 72.68 \\
\hline P3 & 20.93 & 298.55 & 964.96 & 6.93 & 0.09 & 141.55 & 69.41 & 4.04 & 34.48 & 119.87 \\
\hline P12 & 9.95 & 207.43 & 901.39 & 2.49 & 0.17 & 30.20 & 24.70 & 2.26 & 15.60 & 51.33 \\
\hline P13 & 17.01 & 353.84 & 1073.70 & 3.34 & 4.31 & 90.51 & 31.00 & 35.42 & 18.09 & 53.38 \\
\hline P14 & 6.44 & 293.03 & 913.01 & 3.17 & 10.55 & 37.28 & 45.82 & 17.55 & 41.44 & 127.67 \\
\hline P15 & 14.86 & 567.81 & 765.86 & 1.06 & 13.71 & 25.28 & 39.50 & 9.21 & 28.91 & 100.88 \\
\hline P16 & 12.42 & 128.21 & 953.73 & 2.06 & 0.64 & 150.97 & 40.13 & 9.17 & 24.16 & 87.19 \\
\hline $\begin{array}{l}\text { MVP Bistrița- } \\
\text { Năsăud }\end{array}$ & $13.60 \mathrm{~b}$ & $308.14 a$ & $928.77 \mathrm{~b}$ & $3.18 \mathrm{a}$ & $4.91 \mathrm{c}$ & $79.30 \mathrm{~b}$ & $41.76 \mathrm{c}$ & $12.94 \mathrm{a}$ & $27.11 \mathrm{c}$ & $90.05 \mathrm{c}$ \\
\hline SD & 5.16 & 149.90 & 100.47 & 2.01 & 5.89 & 56.98 & 15.46 & 12.23 & 9.85 & 32.48 \\
\hline P5 & 10.33 & 318.05 & 857.71 & 2.89 & 10.65 & 101.85 & 23.63 & 4.53 & 9.27 & 37.19 \\
\hline P7 & 8.39 & 115.60 & 504.18 & 2.06 & 3.16 & 87.46 & 96.66 & 1.69 & 31.47 & 111.96 \\
\hline P8 & 8.53 & 110.95 & 999.81 & 3.06 & 3.38 & 90.54 & 38.59 & 12.46 & 19.72 & 81.78 \\
\hline P9 & 24.11 & 255.02 & 1256.41 & 10.48 & 0.19 & 134.72 & 120.06 & 2.27 & 46.30 & 129.45 \\
\hline P10 & 21.15 & 414.58 & 450.50 & 0.00 & 50.32 & 31.68 & 71.71 & 20.43 & 47.08 & 184.61 \\
\hline P11 & 15.95 & 367.09 & 954.12 & 1.02 & 7.42 & 119.80 & 36.85 & 15.31 & 19.76 & 77.53 \\
\hline $\begin{array}{c}\text { MVP } \\
\text { Maramureş }\end{array}$ & $14.74 a$ & $263.55 b$ & $837.12 \mathrm{c}$ & $3.25 \mathrm{a}$ & $12.52 \mathrm{a}$ & $94.34 \mathrm{a}$ & $64.58 \mathrm{~b}$ & $9.45 \mathrm{c}$ & $28.93 \mathrm{~b}$ & $103.75 b$ \\
\hline SD & 6.76 & 127.87 & 308.78 & 3.73 & 18.87 & 35.56 & 38.15 & 7.75 & 15.45 & 50.73 \\
\hline MVP & $14.49 \mathrm{c}$ & $285.29 b$ & $955.17 \mathrm{~b}$ & $3.09 \mathrm{~b}$ & $8.33 b$ & $89.50 \mathrm{~b}$ & $60.83 a$ & $11.04 \mathrm{~b}$ & $30.45 b$ & $112.72 b$ \\
\hline SD & 6.62 & 157.61 & 221.23 & 2.52 & 11.78 & 46.58 & 33.90 & 8.76 & 13.98 & 56.42 \\
\hline
\end{tabular}

MV-mean value; SD-standard deviation. The values with different superscript letters $(a, b, c)$ are significantly different $(\mathrm{P}<0.05)$

concentrations in plums. The plum brandy is well flavored due to a mixture of acetaldehyde, ethyl acetate and amyl alcohols, mainly responsible for the flavor and its quality depends significantly on their concentration (Apostolopoulou et al., 2005).

For iso-butylic alcohol, amyl active alcohol, iso-amylic alcohol the MVP Cluj values were significantly higher than MVP Bistrița-Năsăud and Maramureş, while for ethyl ac- etate and furfural, the MVP Bistrița-Năsăud were significantly higher than MVP Cluj and MVP Maramureş.

All the present data are in agreement with the recent literature reports (Tab. 9).

To evaluate the differences between the mean values of volatiles in different types of brandies it has been considered the statistical differences between plum-MVP (Tab. 5), apple-MVA (Tab. 6) and pear-MVPe (Tab. 7). For ethyl

Tab. 6. The major volatile compounds found in apple brandy as determined by gas-chromatography (GC-FID)

\begin{tabular}{ccccccccccc}
\hline \multirow{2}{*}{$\begin{array}{c}\text { Sample } \\
\text { code }\end{array}$} & \multicolumn{7}{c}{ Volatile compound mg/100 ml anh. alc. } \\
\cline { 2 - 11 } & Acetaldehyde & $\begin{array}{c}\text { Ethyl } \\
\text { Acetate }\end{array}$ & Methanol & Furfural & 2-Butanol & 1-Propanol & $\begin{array}{c}\text { Iso-butylic } \\
\text { Alcohol }\end{array}$ & $\begin{array}{c}\text { 1-Butanol } \\
\text { Amyl Active } \\
\text { Alcohol }\end{array}$ & $\begin{array}{c}\text { Iso-amylic } \\
\text { Alcohol }\end{array}$ \\
\hline M19 & 13.25 & 231.84 & 597.64 & 1.97 & 0.37 & 21.89 & 44.33 & 14.91 & 39.61 & 146.84 \\
M20 & 18.63 & 473.50 & 1189.29 & 3.06 & 0.39 & 21.42 & 56.68 & 8.26 & 50.56 & 196.59 \\
M21 & 14.98 & 215.68 & 1020.89 & 6.87 & 4.39 & 24.87 & 43.07 & 4.99 & 44.12 & 166.43 \\
M22 & 12.88 & 63.15 & 810.97 & 2.74 & 0.31 & 136.18 & 43.33 & 4.37 & 20.15 & 75.28 \\
MVA & $14.93^{\mathrm{b}}$ & $246.04^{\mathrm{c}}$ & $904.70^{\mathrm{c}}$ & $3.66^{\mathrm{a}}$ & $1.36^{\mathrm{c}}$ & $51.09^{\mathrm{c}}$ & $46.86^{\mathrm{c}}$ & $8.13^{\mathrm{c}}$ & $38.61^{\mathrm{a}}$ & $146.29^{\mathrm{a}}$ \\
SD & 2.63 & 169.62 & 256.62 & 2.19 & 2.01 & 56.75 & 6.57 & 4.83 & 13.10 & 51.57 \\
\hline
\end{tabular}

MV-mean value; SD-standard deviation. The values with different superscript letters $(a, b, c)$ are significantly different $(\mathrm{P}<0.05)$ 
114

Tab. 7. Major volatile compounds found in pear brandy as determined by gas-chromatography (GC-FID)

\begin{tabular}{|c|c|c|c|c|c|c|c|c|c|c|}
\hline \multirow{2}{*}{$\begin{array}{l}\text { Sample } \\
\text { code }\end{array}$} & \multicolumn{10}{|c|}{ Volatile compound $\mathrm{mg} / 100 \mathrm{ml}$ anh. alc. } \\
\hline & Acetaldehyde & $\begin{array}{c}\text { Ethyl } \\
\text { Acetate }\end{array}$ & Methanol & Furfural & 2-Butanol & 1-Propanol & $\begin{array}{c}\text { Iso-butylic } \\
\text { Alcohol }\end{array}$ & 1-Butanol & $\begin{array}{c}\text { Amyl Active } \\
\text { Alcohol }\end{array}$ & $\begin{array}{c}\text { Iso-amylic } \\
\text { Alcohol }\end{array}$ \\
\hline PE23 & 26.09 & 203.52 & 973.61 & 4.69 & 44.80 & 34.66 & 63.79 & 9.51 & 29.67 & 117.59 \\
\hline PE24 & 15.83 & 388.87 & 818.34 & 1.51 & 104.70 & 212.20 & 52.51 & 8.53 & 20.23 & 71.05 \\
\hline PE25 & 27.71 & 136.32 & 880.69 & 2.56 & 19.58 & 59.01 & 59.93 & 17.16 & 41.31 & 145.48 \\
\hline PE26 & 10.04 & 521.75 & 1290.06 & 1.96 & 75.29 & 161.72 & 36.28 & 12.59 & 19.16 & 94.87 \\
\hline MVPe & $19.92^{\mathrm{a}}$ & $312.62^{\mathrm{a}}$ & $990.68^{a}$ & $2.68^{c}$ & $61.09^{\mathrm{a}}$ & $116.90^{\mathrm{a}}$ & $53.13^{b}$ & $11.95^{\mathrm{a}}$ & $27.59^{c}$ & $107.25^{c}$ \\
\hline $\mathrm{SD}$ & 8.43 & 175.63 & 209.54 & 1.41 & 36.93 & 84.07 & 12.17 & 3.88 & 10.29 & 31.79 \\
\hline
\end{tabular}

MV-mean value; SD-standard deviation. The values with different superscript letters $(\mathrm{a}, \mathrm{b}, \mathrm{c})$ are significantly different $(\mathrm{P}<0.05)$

acetate, methanol, 2-butanol, 1-propanol and 1-butanol the MVPe values were significantly higher than MVA. For furfural, amyl active alcohol and iso-amylic alcohol the MVA values were significantly higher than MVPe. For acetaldehyde the MVPe values significantly higher than MVP. Also, in this case, the present data are in agreement with the reported data (Tab. 9).

Tab. 8. The values of the coefficients of correlation between the mean concentrations of methanol-furfural and methanolethanol in the plum, apple and pear brandies

\begin{tabular}{ccc}
\hline Sample & $\begin{array}{c}r \text { values } \\
\text { (methanol-furfural) }\end{array}$ & $\begin{array}{c}r \text { values } \\
\text { (methanol-ethanol) }\end{array}$ \\
\hline Plum brandy & +0.296 & -0.057 \\
Apple brandy & +0.280 & +0.335 \\
Pear brandy & -0.049 & -0.785
\end{tabular}

\section{Correlations between methanol, furfural and ethanol} concentration

Based on the mean values of methanol, furfural and ethanol concentrations, there were calculated the correlation coefficients (Pearson's correlation coefficient), for each type of brandy, considering the relations between methanol release, ethanol and furfural as markers of carbohydrate fermentation and thermal degradation (Tab. 8).

Positive, but no significant correlations between methanol and furfural concentrations in plum brandy and apple brandy, while slight negative correlations (non significant as well) for methanol versus ethanol in pear and plum brandies were registrated. For apple brandy, a positive correlation was noticed between methanol and ethanol, which can be related to higher concentration of methoxylated apple pectins and their degradation to methanol.

Tab. 9. A comparison between the mean values obtained in this study and other reported researches

\begin{tabular}{|c|c|c|c|}
\hline \multirow{2}{*}{$\begin{array}{l}\text { Major volatile } \\
\text { compound }\end{array}$} & \multirow{2}{*}{$\begin{array}{l}\text { Type of fruit } \\
\text { distillate }\end{array}$} & $\begin{array}{l}\text { Mean values obtained } \\
\text { in the present study }\end{array}$ & Values obtained in other reported study \\
\hline & & \multicolumn{2}{|r|}{$\mathrm{mg} / 100 \mathrm{ml}$ anh. alc. } \\
\hline \multirow{3}{*}{ Acetaldehyde } & Plum brandy & $14.49 \pm 6.62$ & 2.6-38.5 (Winterová et al., 2008); 12.4-24.6 (Satora and Tuszyński, 2010) \\
\hline & Apple brandy & $14.93 \pm 2.63$ & 3.0-26.0 (Winterová et al., 2008) \\
\hline & Pear brandy & $19.92 \pm 8.43$ & 1.3-56.2 (Winterová et al., 2008) \\
\hline \multirow{3}{*}{ Ethyl Acetate } & Plum brandy & $285.29 \pm 157.61$ & 56.3-235.9 (Winterová et al., 2008); 27.7-192.5 (Satora and Tuszyński, 2010) \\
\hline & Apple brandy & $246.04 \pm 169.62$ & 12.5-233.4 (Winterová et al., 2008); 17-128 (Versini et al., 2009) \\
\hline & Pear brandy & $312.62 \pm 175.63$ & 7.6-293.7 (Winterová et al., 2008) \\
\hline \multirow{3}{*}{ Methanol } & Plum brandy & $955.17 \pm 221.23$ & 287.7-1141.4 (Winterová et al., 2008); 755-974.4 (Satora and Tuszyński, 2010) \\
\hline & Apple brandy & $904.70 \pm 256.62$ & 179.4-916.8 (Winterová et al., 2008); 540-1140 (Versini et al., 2009) \\
\hline & Pear brandy & $990.68 \pm 209.54$ & 93.2-1080.9 (Winterová et al., 2008) \\
\hline \multirow{3}{*}{ Furfural } & Plum brandy & $3.09 \pm 2.52$ & \\
\hline & Apple brandy & $3.66 \pm 2.19$ & 0.23-6.23 (Versini et al., 2009) \\
\hline & Pear brandy & $2.68 \pm 1.41$ & \\
\hline \multirow{3}{*}{ 1-Propanol } & Plum brandy & $89.50 \pm 46.58$ & 35.6-308.4 (Winterová et al.,2008) \\
\hline & Apple brandy & $51.09 \pm 56.75$ & 12.1-229.0 (Winterová et al., 2008); 15.0-73 (Versini et al., 2009) \\
\hline & Pear brandy & $116.90 \pm 84.07$ & 14.1-706.8 (Winterová et al., 2008) \\
\hline \multirow{3}{*}{ 1-Butanol } & Plum brandy & $11.04 \pm 8.76$ & 2.1-12.6 (Winterová et al., 2008) \\
\hline & Apple brandy & $8.13 \pm 4.83$ & 8.0-20.5 (Winterová et al., 2008), 6.3-116 (Versini et al., 2009) \\
\hline & Pear brandy & $11.95 \pm 3.88$ & 1.6-22.8 (Winterová et al., 2008) \\
\hline \multirow{3}{*}{ 2-Butanol } & Plum brandy & $8.33 \pm 11.78$ & 1.3-19.5 (Winterová et al., 2008) \\
\hline & Apple brandy & $1.36 \pm 2.01$ & 0.8-32.3 (Winterová et al., 2008) \\
\hline & Pear brandy & $61.09 \pm 36.93$ & 0.6-73.3 (Winterová et al., 2008) \\
\hline
\end{tabular}


For acetaldehyde, ethyl acetate, methanol and all superior alcohols pear brandy samples registered significantly higher values than plum or apple; only furfural had the lowest value for pears, apple brandy being the richest in furfural (Tab. 5-7).

Methanol represents the most important volatile component, which, as described before was released during processing, and it is characteristic to fruit brandies, significantly higher than in cereal distillates, due to methoxylated pectin degradations. Therefore, this molecule can be considered not only a parameter of distillate safety but also may indicate, indirectly, the natural origin and the fermentation technology used in traditional processing.

Anyway, in all 26 samples of fruit brandies the values of methanol concentrations were below the maximum admissible values $(1200 \mathrm{mg} / 100 \mathrm{ml}$ anh.alc) established by European Commission.

\section{Conclusions}

Using GC-FID analysis three different types of brandies (apple, pear and plum) originating from Transylvania region, Romania, were compared. The volatiles identified and quantified using GC-FID analysis were: acetaldehyde, ethyl acetate, methanol, 2-butanol, 1-propanol, iso-butilic alcohol, 3-pentanol (internal standard), 1-butanol, amyl active alcohol, iso-amylic alcohol and furfural. Their average values were in concordance with other reported data from other European countries (Czech Republic, Slovenia, Serbia, Italy, Greece, and Turkey). Acetaldehyde, ethyl acetate and amyl alcohols are the main responsible for the flavor of distilled beverages, and the quality of these drinks depends on their concentration. Calculations were performed on the correlation coefficients for each type of brandy considering the relations between the methanol release, ethanol and furfural, as a marker of carbohydrate thermal degradation. Positive but no significant correlations between methanol and furfural concentrations in plum brandy and apple brandy were also noticed.

To evaluate the differences in composition regarding the geographical origin of plum brandies and to compare the composition of plum, apple and pear brandies, it has been compared the mean values (MVP, MVA and MVPe) obtained for each volatile.

For plum brandies, it has been observed differences among the mean values (MVP) of each volatile, in plum brandies originating from 3 counties, Cluj, Bistrița-Năsăud and Maramureş, Further investigations will be focused more on authenticity parameters for these three types of Romanian traditional brandies from Transylvania.

\section{References}

Apostolopoulou AA, Flouros AI, Demertzis PG, AkridaDemertzi K(2005). Differences in concentration of principal volatile constituents in traditional Greek distillates. Food
Control 16(2):157-164.

Beceanu D, Niculaua M (2009). A comparative study of an assortment of plum distilled drinks, made in Romania. Agronom Res in Mold 139(3):49-61.

Beceanu D, Niculaua M, Moraru I, Anghel RM (2010). Studies regarding the quality of certain fruit distillates in correlation with the analytical data and sensorial assessment. Agronom Res Mold 144 (4):61-77.

Berglund KA (2004). Artisan Distilling. A guide for small distilleries. Electronic Edition, http://www.aec.msu.edu/ product/documents/ARTISANDISTILLING1.0.01.pdf.

Christopoulou-Gerogiannaki M, Gerogiannaki I, Anagnostartas E, Stavrakas DE, Polissiou M (2007). Identification of volatile compounds in Hellenic alcoholic beverages from native white grape varieties (Vitis vinifera L.). J Food Technol 5(3):233-241

Dragone G, Mussatto SI, Oliveira JM, Teixeira JA (2009). Characterisation of volatile compounds in an alcoholic beverage produced by whey fermentation. Food Chem 112:929-935.

Ertan Anli R, Vural N, Gucer Y (2007). Determination of the principal volatile compounds of Turkish Raki. J Inst Brew 113(3):302-309.

García Llobodanin L (2008). Potencial of Blanquilla pear variety to produce pear spirits: Influence of the fermentation and distillation conditions in the final quality. Universitat Rovira i Virgili, Tarragona, Spain, PhD Diss.

Geroyiannaki M, Komaitis ME, Stavrakas DE, Polysiou M, Athanasopoulos PE, Spanos M (2007). Evaluation of acetaldehyde and methanol in greek traditional alcoholic beverages from varietal fermented grape pomaces (Vitis vinifera L.). Food Control 18:988-995.

Korhonova M, Hejdova R, Cap L, Lemr K, Bednar P, Bartak P (2006). GC-MS analysis of markers for characterization of distillates. 17th International Mass Spectrometry Conference, Prague.

Martínez Montero C (2006). A study of alternative parameters as indicators of aging and quality for brandy de Jerez (in Spanish). Universidad de Cádiz, PhD Thesis.

Moales D, Spac AF, Prisecaru M, Butnaru E (2010). Determining the concentration of methanol from natural distillate. Sci Res Biol 19:51-60.

Modig T, Lidén G, Taherzadeh MJ (2002). Inhibition effects of furfural on alcohol dehydrogenase, aldehyde dehydrogenase and pyruvate dehydrogenase. Biochem J 363:769-776.

Nikićević N, Tešević V (2005). Possibilities for methanol content reduction in plum brandy. J Agricult Sci 50(1):49-60.

Pomohaci N, Cioltean I, Vişan L, Rădoi F (2002). Tuica and natural brandies (in Romanian). Ed. Ceres, Bucharest.

Popović B, Gavrilović-Damnjanović J, Mitrović O, Ogasanović D, Nikićević N, Tesević V (2009). Major volatile components and sensory characteristics of plum brandies produced from plum cultivars developed in Čačak. Acta Horti 825:575- 
116

581.

Quesada Granados J, Villalón Mir M, López García-Serrana H, López Martínez MC (1996). Influence of aging factors on the furanic aldehyde contents of matured brandies: aging markers. J Agric Food Chem 44:1378-1381.

Regulation 110/2008 of the European Parliament and of the Council. 15 January 2008 on the definition, description, presentation, labelling and the protection of geographical indications of spirit drinks and repealing. Council Regulation (EEC) No 1576/89. Official Journal of the European Union 51(39):16-54.

Satora P, Tuszyński T (2010). Influence of indigenous yeasts on the fermentation and volatile profile of plum brandies. Food Microbiol 27:418-424.

Tešević V, Nikicevic N, Jovanovic A, Djokovic D, Vujisic L, Vuckovic I, Bonic M (2005). Volatile components from old plum brandies. Food Technol Biotechnol 43(4):367-372.
Tešević V, Nikićević N, Milosavljević S, Bajić D, Vajs V, Vučković I, Vujisić L, Đorđević I, Stanković M, Veličković M (2009). Characterization of volatile compounds of "Drenja", an alcoholic beverage obtained from the fruits of cornelian cherry. J Serb Chem Soc 74(2):117-128.

Tóthová J, Žiak L', Sádecká J (2008). Characterization and classification of distilled drinks using total luminescence and synchronous fluorescence spectroscopy. Acta Chim Slov $1(1): 265-275$.

Versini G, Franco MA, Moser S, Barchetti P, Manca G (2009). Characterisation of apple distillates from native varieties of Sardinia island and comparison with other Italian products. Food Chem 113:1176-1183

Winterová R, Mikulíková R, Mazáč J, Havelec P (2008). Assessment of the authenticity of fruit spirits by gas chromatography and stable isotope ratio analyses. Czech J Food Sci 26(5):368-375. 